\title{
Transgenic Approaches of Improving Tomato (Solanum lycopersicum) to Salt Stress Tolerance
}

\author{
Mengistu Wubie Birhanu \\ Department of Horticulture, College of Agriculture and Natural Resources, Debre Markos University, \\ P. O. Box 269, Debre Markos, Ethiopia.
}

\begin{abstract}
Environmental stresses mainly drought and salinity are now becoming potential threats for global agricultural production. Being a tropical crop, tomato is produced in warm and dry regions of the world, where salt stress is limiting the yield potential of the crop. Plant defense response to salt tolerance is very complex in which many morphological, physiological, and molecular modifications are enhanced. Although considerable effort has been made in developing salt tolerant tomato cultivars through conventional breeding, it has met with limited success due to the complex, multigenic nature of the trait and developmental stage regulated phenomenon of the salt tolerance response. Genetic engineering is an alternative approach to understand the mechanisms of salinity tolerance and apply the knowledge obtained to generate salt tolerant tomatoes. This review aims to highlight the latest developments in biotechnological techniques of improving tomato tolerance to salt stress. Recently, there have been many attempts to enhance tomato tolerance to salt stress including introduction and/or modifications of various genes involved in regulatory, signaling pathways, detoxifying enzymes and genes encoding functional and structural proteins. Plasma membrane $\mathrm{Na}^{+} / \mathrm{H}^{+}$antiporters (SOS) and vacuolar $\mathrm{Na}^{+} / \mathrm{H}^{+}$antiporters $(\mathrm{NHXn})$, Dehydrin (DHN), Choline Oxidase ( $\operatorname{codA})$, and ROS scavengers have been the major genes targeted in many studies for developing salt stress tolerant transgenic tomato. Moreover, promising results have reported on the identification and use of various abiotic stress responsive elements and/or transcription factors such as SIDREB2, SL-ZH13, Sl-ERF.B.3, AREB, NAC-Type and WRKY TFs, which positively regulate the expression of the downstream genes in transgenic tomato with response to salt stress.
\end{abstract}

Keywords: Salt Stress, Tomato, Transgenic, Transcription Factor

DOI: $10.7176 / \mathrm{JBAH} / 10-14-01$

Publication date:July $31^{\text {st }} 2020$

\section{Introduction}

Environmental stresses especially drought and salinity are now becoming potential threats for global agricultural production. About $70 \%$ of crop yield reduction is estimated due to environmental stresses (Acquaah, 2009). The severity of such environmental stresses becomes more serious when two or more types of stresses occur simultaneously. Scarcity of fresh water for irrigated agriculture might be a future challenge as the demand of food production increased with human population (Yadav et al., 2013).

Tomato (Solanum lycopersicum) belongs to Solonacea family and is one of the most important vegetable crops, which is produced for the fresh market and processed products such as tomato juice, paste, puree, ketchup, sauce, and salsa. It is a major source of minerals, vitamins, and provides health benefits in human consumption (Robertson and Labate, 2007). The tomato crop has wide climate adaptability ranging from the tropics to a few degrees of the Arctic regions. Despite its economic value, the productivity of tomato is influenced by certain biotic and abiotic factors. Though grown in a wide range of areas, the major share of tomato production is mainly concentrated in a warm and dry regions including Mediterranean Sea, Southern and Western parts of USA and Mexico (Foolad, 2004). However, due to frequent occurrence of soil and/or water salinity, the production of tomato in such warm and dry areas is constrained by salt stress.

Most commercial tomato cultivars are sensitive or moderately sensitive to salt stress. Salt stress reduces leaf fresh and dry weight, leaf area, leaf number and chlorophyll content in tomato (Rebah et al., 2018). Yield reduction in tomato due to salt stress is mainly related to reduction in fruit weight and number of fruit under low and severe salinity stress, respectively (Cuartero and Fernández-Muñoz, 1998). On the other hand, it has been reported that salinity enhances some quality parameters such as lycopene, total anthocyanins, and increased the differences in color of different purees (Borghesi et al., 2011). This suggests the possibility of improving the synthesis of secondary metabolites including carotenoids and anthocyanins by cultivating tomato in salt affected soil. Tolerance or sensitivity of tomato to salt stress depends on the life cycle and developmental stage of the crop though most studies are conducted at germination and early growth stage of the crop (Foolad, 2004). Thus, it is advisable that researches should focus on at vegetative stage and reproductive stage since tomato is grown as transplanting seedlings rather than direct sowing in the field.

The adverse effects of salinity stresses could be alleviated by different management aspects such as fresh water irrigation and remediation of salt affected soil. However, in developing countries, where these technologies are not easily available and expensive, development of resistant cultivars to salt stresses is an economic and 
efficient method to increase crop productivity (Ashraf and Wu, 1994). Therefore, researches are needed to identify and deploy tolerance mechanisms that will allow crops to maintain productivity in adverse environment. Although considerable effort has been made in developing salt tolerant tomato cultivars through conventional breeding, it has met with limited success due to the complex, multigenic nature of the trait and the developmental stage regulated phenomenon of the salt tolerance response (Cuartero et al., 2006). Therefore, genetic engineering is an alternative approach to both understand the mechanisms of salinity tolerance and apply the knowledge obtained to generate salt tolerant tomatoes. Several genes have been transformed in tomato to improve salt tolerance and promising results have been reported.

The objective of this review is to highlight the latest developments in the field of biotechnological techniques of improving salt stress tolerance in tomato. The review focus on salinity as a global challenge to agricultural production, response mechanisms of crop plants to salinity, the role of plant growth regulators (phytophormones) in salinity tolerance in cop plants, the different approaches used and various types of genes cloned and transferred in tomato for improving salt stress tolerance.

\section{Salt Stress as a Global Threat to Agricultural Crop Production}

The world food production must increase largely to ensure food security for the over growing population. However, food production is seriously threatened by various environmental factors. Salinization of soil and/or water is one of the most serious environmental stresses, particularly in arid and semi-arid regions, causing huge yield loss in many cultivated crops throughout the world. Salinity affects more than 800 million ha of land worldwide (Rengasamy, 2010). And the problem of salinity is now becoming more serious due to poor agricultural practice and other related problems. In general, there are two major causes of salinity i.e. natural processes and humanaided. In arid and semiarid regions, most of the salt affected lands are due to the accumulation of salts in soil or water surface overtime from weathering of parental rocks containing soluble salts of $\mathrm{Na}^{+}, \mathrm{Mg}^{2+}$, and $\mathrm{Ca}^{2+}$ chlorides (Szabolcs, 1989). The deposition of oceanic salt carried in wind and rain is another cause of natural salinity. Salinity might also occur from the groundwater. When there is high accumulation of salt ion in the ground water, it moves upward through capillary force and remains at the soil surface while water is evaporated. Moreover, significant amount of cultivated land is also becoming saline due to human practice such as land clearing and irrigation. More than $20 \%$ of irrigated land in the world is salt affected and the trend is increasing due to climate change and excessive application of irrigation water (Hasanuzzaman et al., 2013).

Salinity affects almost every aspect of the physiology and biochemistry of plants and causes significant yield reduction (Foolad, 2004). The detrimental effects of salinity are mainly reduction of water potential resulting in osmotic stress, $\mathrm{Na}^{+}$and $\mathrm{Cl}^{-}$toxicity on physiological and biochemical process of plants, and disruption of nutrient availability (Munns, 2002). In moderately saline condition, plants are facing difficulty for absorbing water hence low water absorption causes wilting, slow growth, and finally yield reduction. Plants maintain lower water potential to uptake water for growth and cell turgidity. However, as the salt concentration of the soil increases and water potential decrease, water moves from the root cells into the surrounding soil solution due to concentration gradient so that plants suffer stress due to loss of water from their tissues. In general, the major effects of salinity on plants includes altered water uptake, inhibition of germination, growth retardation, inhibition of photosynthesis, oxidative stress, and ultimately yield reduction (Hasanuzzaman et al., 2013). Except for the biochemical disturbances, morphological alterations, such as reduction in leaf area, stomata closure, and reduction in stomata conductance, reduce the rate of photosynthesis.

Thus, the significant effect of salinity on agricultural productivity has to be tackled either by improving agricultural practices and/or remediating salt affected land. However, developing salt tolerant cultivars through traditional breeding and/or genetic engineering approach seems the best strategy from sustainable management perspectives. To do so it is crucial that the morphological, physiological, and molecular response of plants to salt stress is well understood.

\section{Mechanisms of Salt Tolerance in Plants}

Plant defense response to salt tolerance is very complex in which many morphological, physiological, and molecular modifications are enhanced. Plants have different mechanisms to tolerate high level of salt. Morphological response is the first adaptive mechanism of plants under salt stress. Similar to drought, salt stress limits photosynthesis due to closure of stomata and low $\mathrm{CO}_{2}$ intake. Hence, slow growth rate is an adaptive response which enables plants to save energy and combat stress (Zhu, 2001). In Arabidopsis, the DELLA proteins are reported to hinder plant growth under adverse environment by integrating hormonal signals (Achard et al., 2006). Thus, smaller plants survive better under stress conditions because of less surface area and efficient resource allocations. Reduced in stomatal density which enhance low transpiration rate allows plants to tolerate high salinity. Low stomatal density and reduction of transpiration rate in salt tolerant strawberry cultivar suggest low accumulation of toxic ions in the leaves, reducing dehydration and osmotic adjustment (Orsini et al., 2012).

Ion exclusion and inclusion are the two main types of tolerance mechanisms of plants to survive in saline 
condition (Munns, 2002). Salt excluders, most glycophytes, restrict salt ion movement to the shoot by controlling ion influx at the root xylem. Whereas, halophytes (salt includers) uptake high salt ions and accumulate in intracellular or vacuolar compartment to reduce the toxic effect of $\mathrm{Na}^{+}$as sodium is mainly accumulates in transpiration stream of the leaf blade part. The capacity of plants to maintain high $\mathrm{K}^{+} / \mathrm{Na}^{+}$by excluding or restricting entrance of $\mathrm{Na}^{+}$in to the cells and/or compartmentalization of $\mathrm{Na}^{+}$in the vacuoles is one of the key strategy of plants to maintain ion homeostasis.

$\mathrm{Na}^{+}$enters to the root cells passively as water moves through the root cortex or with the involvement of $\mathrm{Na}^{+}$ transporters (Munns and Tester, 2008). The Arabidopsis $\mathrm{Na}^{+}-\mathrm{H}^{+}$co- transporter AtHKT1 has shown as a mediator in selective $\mathrm{Na}^{+}$transport and to some extent $\mathrm{K}^{+}$transport (Uozumi et al., 2000). Its expression was higher in roots while low transcript has observed in flowers, leaves and stem parts. Even though AtHKT1 is known in $\mathrm{Na}^{+}$influx to plant cells, its members such as AtHKT1;1 have shown to be involved in reducing $\mathrm{Na}^{+}$influx to the shoot parts by removing $\mathrm{Na}^{+}$from the transpiration stream (Munns and Tester, 2008). Once $\mathrm{Na}^{+}$entered to the transpiration stream of root, it transported to the shoot parts through xylem vessels. Thus, one attribute of plants to tolerate long term effect of salinity is exclusion of $\mathrm{Na}^{+}$from the root, transpiration stream or from the shoot parts. The SOS (Salt Overly Sensitive) signaling pathway suggested having critical role in $\mathrm{Na}^{+}$exclusion from roots and maintaining ion homeostasis (Shi et al., 2002b). Not only from the roots the plasma membrane SOS has also significant role in $\mathrm{Na}^{+}$exclusion from the transpiration stream and shoots. The SOS1-overexpression transgenic Arabidopsis plants showed low level of $\mathrm{Na}^{+}$concentration in the xylem sap compared to control plants after one day of $100 \mathrm{mM} \mathrm{NaCl}$ treatment (Shi et al., 2002a). In another study, high accumulation of $\mathrm{Na}^{+}$in stem tissue rather than leaf and no senescence after high salt treatment in tolerant potato cultivars suggest stem tissues have a role in salt accumulation in resistant plants (Jaarsma et al., 2013).

Vacuolar compartmentalization of $\mathrm{Na}^{+}$is a principal mechanism of plants to reduce $\mathrm{Na}^{+}$toxicity and osmotic effect of high salinity. The vacuolar membrane $\mathrm{Na}^{+} / \mathrm{H}^{+}$antiporter $\mathrm{NHX} 1$ plays crucial role in transporting $\mathrm{Na}^{+}$into the vacuoles and reduce ion toxicity (Sottosanto et al., 2007). The role of the vacuolar $\mathrm{Na}^{+} / \mathrm{H}^{+}$transporter NHX1 in salt tolerance has been confirmed in different plant species including Arabidopsis, Beta vulgaris, and tomato (Apse et al., 1999; Xia et al., 2002; Zhang and Blumwald, 2001).

The other most important salt tolerance mechanism of plants is increasing the synthesis of compatible solutes or osmolytes; organic compounds such as sucrose, proline, and glycine betaine to balance the osmotic pressure (Munns and Tester, 2008). Thus, these compounds prevent water efflux from the plant cell, maintain cell expansion, and reduce oxidative stress to make plants tolerant to salt stress. In salt tolerant potato cultivars, the concentration of proline and enzymes responsible for synthesis of proline, P5CS1 (Delta 1-pyrroline-5-carboxylate synthase) and P5CR (Delta 1-pyrroline-5-carboxylate reductase), have been increased when treated with high concentration of $\mathrm{NaCl}$ (Jaarsma et al., 2013). Moreover, significant reduction in proline catabolism gene expression, PDH (proline dehydrogenase) in leaf and stem suggests the correlation between P5CR concentration and PDH expression.

When plants are exposed to certain environmental stresses including salt stress, the formation of reactive oxygen species (ROS) such as hydroxyl radical, hydrogen peroxides, singlet oxygen $\left({ }^{1} \mathrm{O}_{2}\right)$, and superoxide radical are enhanced. Under salt stress, the imbalance between photosynthetic light capture and utilization of the fixed $\mathrm{CO}_{2}$ changes the redox state causing photo-inhibition, which led to the formation of highly toxic ROS. ROS cause oxidative damage such as enzyme inactivation, lipid peroxidation, and protein damage.

Plants have developed antioxidant defense system to protect cells from oxidative injury. Enzymatic and nonenzymatic antioxidants are involved in scavenging active oxygen species and reduce oxidative stress (Vranova et al., 2002). Superoxide dismutase (SOD), peroxidase (POX), glutathione reductase (GR), catalase (CAT), and ascorbate peroxidase (APX) are the most important antioxidants in scavenging ROS (Yang et al., 2013). In wheat, the enhancement of catalase, peroxidase and ascorbate peroxidase, and glutathione reductase activities under salt stress tolerant cultivar suggest the involvement of these enzymes in detoxifying reactive oxygen species to reduce damage to cell membrane (Mandhania et al., 2006).

\section{Phytohormones in Salinity Tolerance}

Plant hormones such as ethylene (ET), salicylic acid (SA), gibberellins (GA), jasmonic (JA), and abscisic acid (ABA) are naturally occurring endogenous substances involved in plant growth and development, fruit ripening, and regulating various stress related processes. Phytohormonal activity induces alteration of morphological and physiological process at cellular and molecular level that enables plants to survive under stress conditions.

Besides to regulating key processes related to seed germination and plant development, ABA is considered as major stress hormone because of its rapid accumulation and playing pivotal role in response to biotic and abiotic stresses (Lee and Luan, 2012). Upon stress, ABA regulates water status of plants by regulating opening and closure of stomata. In soybean, the level of ABA in leaves significantly increased with increasing salt concentration. This increment in ABA caused stomata closure and lower photosynthesis which finally led to hindering plant growth. Its level is controlled by the balance between ABA biosynthesis and ABA catabolism. The 9-cis- epoxycarotenoid 
dioxygenase (NCED) play key role in ABA biosynthesis. In Arabidopsis, the AtNCED3 gene was highly up regulated under drought condition and the disruption of AtNCED3 caused decrease in ABA level which results in high transpiration rate in AtNCED3 mutants Arabidopsis plants (Iuchi et al., 2001).

It has been reported that exogenous application of phytohormones induces tolerance in response to salinity. Exogenous application of $100 \mathrm{uM} \mathrm{ABA}$ before and during salt stress enhanced salt tolerance in both salt susceptible and resistant rice cultivar by inducing OsP5CS1 gene expression (Sripinyowanich et al., 2010). Moreover, the expression of calmodulin gene (OsCam1-1) was up-regulated in resistant rice cultivar. Thus, the expression of OsP5CS1 and OsCam1-1 suggests high accumulation of proline as osmo-protectant. Ethylene is another stress hormone involved in development and biotic and abiotic stress related processes. It acts as membrane receptor, components in cytoplasm, and nuclear transcription factor in signaling pathways. Gibberellin is another plant growth hormone regulated by developmental and environmental factors. Application of GA3 in soybean significantly increased plant height, fresh and dry weight under salt stress condition (Hamayun et al., 2010b). Further phytohormonal analysis showed that the level of bioactive gibberellins (GA1 \& GA4) and jasmonic acid was significantly increased, while the endogenous SA and ABA level declined in GA3 treated plants, suggesting GA3 reduce the adverse effect of salt stress by regulating the level of endogenous phytohormones. Similar study showed that salinity reduces growth performance of Arabidopsis through modulating GA metabolism pathways (Achard et al., 2006). In another study, salt stress decreased the endogenous GA and SA, while ABA and JA significantly increased in salt stressed soybean plants (Hamayun et al., 2010a). It was concluded that salinity induce growth retardation and yield reduction in soybean by interfering with endogenous growth hormones.

\section{Transgenic Approach to Improve Tomato Salt Tolerance}

Several studies have been conducted for the enhancement of salt tolerance in transgenic tomato through either overexpression of endogenous or foreign genes that are supposed to be involved in the process of salt tolerance. Recently, attempts have been done to enhance tomato tolerance to salt stress including introduction and/or modifications of various genes involved in ion transport, antioxidant enzymes, regulatory and signaling pathways (Table 1).

\subsection{Genes Encoding Ion Transport Proteins}

Sodium can be sensed by membrane receptor, membrane proteins or by any sodium sensitive enzymes in the cytoplasm. Ion transporters (NHX proteins) have been reported to be important candidates for salt tolerance by promoting accumulation of $\mathrm{Na}^{+}$or $\mathrm{K}^{+}$inside vacuoles and ion homeostasis. One way of biotechnological method of tomato improvement to tolerance stress is modifying genes encoding ion transport proteins. The Arabidopsis vacuolar $\mathrm{Na}^{+} \mathrm{H}^{+}$antiporter (AtNHX1) was the first candidate gene in which the transgenic plants showed higher ability for vacuolar sequestration of $\mathrm{Na}^{+}$to avoid toxic accumulation in the cytoplasm. This was clearly demonstrated in transgenic tomato plants overexpressing $\mathrm{Na}^{+} / \mathrm{H}^{+}\left(\mathrm{AtNHX}_{1}\right)$ from Arabidopsis, which performed better under $200 \mathrm{mM} \mathrm{NaCl}$ as compared to the WT tomato plants (Zhang and Blumwald, 2001). There was no variation in number of fruits per plant. Although the fruits from the transgenic plants grown in $200 \mathrm{mM} \mathrm{NaCl}$ were a bit smaller, no significant difference was observed in their water content or total soluble solids content. Moreover, accumulation of high concentration of salt in the leaves rather than fruit was shown and does not interfere with fruit quality suggesting the potential use of this transgenic tomato in saline soil. Studies have confirmed that differences exist between domesticated and wild- relative species of tomato with regard to salt stress response (Albaladejo et al., 2015). Generally, wild tomato species are suggested to be more salt tolerant than cultivated species mainly due to high accumulation of $\mathrm{Na}^{+}$in aerial parts (Gálvez et al., 2012). This was confirmed as salt stress induced the expression of LeNHX3 and LeNHX4 isoforms in salt tolerant wild species of tomato. The NHX genes enhanced $\mathrm{Na}^{+}$accumulation in aerial parts of the wild tomato species when grown in the presence of $\mathrm{NaCl}$.

Therefore, overexpression of AtNHX1 and related NHX proteins from various sources have been shown to improve salt tolerance in tomato. This has been observed, for instance, in overexpressing wheat $\mathrm{Na}^{+} / \mathrm{H}^{+}$antiporter gene $\left(\mathrm{TaNHX}_{2}\right)$ in tomato (Yarra et al., 2012a). The $\mathrm{TaNHX}_{2}$-overexpression transgenic tomato lines showed better germination rate, growth performance, flowering and set viable seeds under 100 or $150 \mathrm{mM} \mathrm{NaCl}$. Moreover, significantly high leaf water content (LWC) and chlorophyll content has been observed in transgenic tomato lines as compared to WT plants. Similarly, Bhaskaran and Savithramma (2011) demonstrated that co-expression of PgNHX1 and AVP1 genes in tomato improves salt tolerance in transgenic tomato plants. The transgenic lines showed salinity tolerance up to $300 \mathrm{mM} \mathrm{NaCl}$ concentrations, whereas wild-type plants died at 200 to $250 \mathrm{mM}$ $\mathrm{NaCl}$. The result suggests that antiporter PgNHX1and AVP1 effectively sequestered excess $\mathrm{Na}^{+}$and $\mathrm{H}^{+}$into vacuole. Accumulation of high levels of proline maintain a good $\mathrm{K}^{+} / \mathrm{Na}^{+}$ratios, high retention of chlorophyll, soluble sugar and water content during stress, in addition to high germination percentage conferred better tolerance to salinity stress. Other study reported that NHX proteins are candidates for the $\mathrm{H}^{+}$-linked $\mathrm{K}^{+}$transport that is thought to facilitate active $\mathrm{K}^{+}$uptake at the tonoplast, and the partitioning of $\mathrm{K}^{+}$between vacuole and cytosol without necessarily $\mathrm{Na}^{+}$compartmentalization in the vacuole (Leidi et al., 2010). AtNHX1 overexpressing 
transgenic tomato lines had a greater capacity to tolerate different salt concentration compared to control plants. Moreover, $\mathrm{K}^{+}$content was consistently higher in transgenic lines under all growth conditions tested with minor impact on the capacity for $\mathrm{Na}^{+}$sequestration inside vacuoles when plants were subjected to salinity stress. This tolerance mechanism was suggested due to the role of AtNHX1 antiporter in $\mathrm{K}^{+}$homeostasis by capturing $\mathrm{K}^{+}$in the vacuoles, as well as the simultaneous increment of compatible solutes (free sugars and proline) in the cytosol (Leidi et al., 2010).

Most NHXs are essential for $\mathrm{Na}^{+}$detoxification via sequestration of $\mathrm{Na}^{+}$within the vacuole, while the SOS signaling pathway is reported to be involved in exporting $\mathrm{Na}^{+}$out of the cell. The plasma membrane $\mathrm{Na}^{+} / \mathrm{H}^{+}$ antiporter (SOS1) plays significant role in $\mathrm{Na}^{+}$extrusion and in controlling long-distance $\mathrm{Na}^{+}$transport from the root to shoot (Pardo, et al., 2007). Overexpressing SISOS2 improves the growth performance, early flowering, and high yield production compared to non-transformed tomato (Huertas et al., 2012). Overexpression of SISOS2 up regulate the expression of plasma membrane $\mathrm{Na}^{+} / \mathrm{H}^{+}$antiporter (SISOS1) and vacuolar $\mathrm{Na}^{+} / \mathrm{H}^{+}$antiporters (LeNHX2 and LeNHX4). LeNHX2 is previously reported as essential $\mathrm{K}^{+} / \mathrm{H}^{+}$antiporter for normal plant growth and development, and plays an important role in the response to salt stress through improving $\mathrm{K}^{+}$accumulation. Gisbert et al. (2000) reported that the yeast HAL1 gene conferred salt tolerance in transgenic tomato both in vitro and in vivo conditions. The higher $\mathrm{K}^{+} / \mathrm{Na}^{+}$ratio in the transgenic plants suggests $H A L 1$ facilitate the accumulation of high $\mathrm{K}^{+}$and decreasing intracellular $\mathrm{Na}^{+}$concentration under salt stress. This suggests HAL genes reduced the movement of $\mathrm{Na}^{+}$from roots to shoots under salt stress.

\subsection{Genes Encoding Antioxidant \& Other Protective Proteins}

One of the major effects of abiotic stresses including salinity is over accumulation of Reactive Oxygen Species (ROS) such as superoxide $\left(\mathrm{O}_{2}^{-}\right)$, hydrogen peroxide $\left(\mathrm{H}_{2} \mathrm{O}_{2}\right)$, nitric oxide $(\mathrm{NO})$ and $\mathrm{OH}$. ROS are normally produced in different cellular compartments during normal physiological processes and serve as signaling molecules. However, they are highly toxic and cause damage to proteins, lipids, carbohydrates, DNA, and membrane function in plant cells if they are produced in high rate (Gill and Tuteja, 2010). To combat these highly toxic substances, plants have antioxidant response proteins such as superoxide dismutase (SOD), ascorbic peroxide (APX), catalase (CAT) and other non-enzymatic antioxidants. Enzymes such as glyoxalases are involved in detoxification of methylglyoxal. Studies reported that tomato transgenic lines overexpressing glyoxalase I (GlyI) and glyoxalase II (GlyII) genes showed reduced lipid peroxidation and the production of $\mathrm{H}_{2} \mathrm{O}_{2}$ in leaf tissues compared with the WT under $800 \mathrm{mM} \mathrm{NaCl}$ concentration (Viveros et al., 2013). Enhanced tolerance of tomato overexpressing GlyI and GlyII was related to reduction in oxidative stress in transgenic lines. Moreover, the overexpressed transgenic lines showed higher chlorophyll $\mathrm{a}+\mathrm{b}$ as compared to the WT.

Melatonin (N-acetyl-5-methoxytryptamine) is another antioxidant having a powerful role in ROS scavenging activity and improves tolerance to various stresses. The melatonin bio-synthesis related gene (S1COMT1) overexpressing transgenic tomato showed higher melatonin content under $800 \mathrm{mM}$ salt stress (Liu et al., 2019). The level of superoxide and hydrogen peroxide were decreased while proline content was increased in SICOMT1 transgenic tomato plants. Other study reported that transgenic tomato (Solanum lycopersicum cv. 'Moneymaker') with the choline oxidase gene (codA) targeted to both chloroplast and cytosol results high accumulation of glycinebetaine (GB), which leads to alleviate salt-induced potassium efflux. The codA-transgenic tomato plants and exogenously treated WT with GB enhanced salt tolerance by increasing $\mathrm{Na}^{+}$exclusion and decreasing the $\mathrm{K}^{+}$ efflux so as maintaining $\mathrm{Na}^{+} / \mathrm{K}^{+}$homeostasis in salt stress. Moreover, high accumulation of GB in the leaves enhances the activities of antioxidant enzymes, which scavenge ROS and decrease the levels of ROS in leaves, which in turn alleviate the effects of salt stress on the photosynthetic machinery (Wei et al., 2017).

Salt stress tolerance can also be improved through modification of various genes involved in stress response and tolerance. Liu et al. (2015), for instance, demonstrated that overexpression of a dehydrin gene (ShDHN) from Solanum habrochaites improves the growth performance of transgenic tomato seedlings to salt stresses. The result from this study indicated that the growth attributes such as fresh weight, primary root length, and number of lateral roots per seedling of the transgenic tomato lines were significantly higher than the WT under $150 \mathrm{mM} \mathrm{NaCl}$. To further elucidate the molecular mechanisms of stress tolerance mediated by ShDHN, the expression of genes related to ROS generation and scavenging, sugar metabolism, proline and JA biosynthesis pathways, and other stress-responsive genes were analyzed both in ShDHN-overexpressing lines and WT plants grown normal condition. Genes coding for SOD and glutathione S-transferase (GST), were significantly up-regulated, while genes encoding peroxidase and lipoxygenase gene (LOX) was down-regulated in the transgenic lines compared to the WT. Similarly, the overexpression of a dehydrin gene (tas14) improves tomato tolerance to salt and drought stress without any pleiotropic effect (Muñoz-Mayor et al., 2012). The transgenic tomato showed increased $\mathrm{Na}^{+}$ accumulation only in adult leaves, whereas $\mathrm{K}^{+}$and sugars more accumulated in young leaves. This result suggests that the transgenic plants overexpressing tas 14 gene are able to distribute the $\mathrm{Na}^{+}$accumulation between young and adult leaves. The transgenic lines accumulated more ABA in leaves during short-time periods.

Mannitol is a type of sugar alcohol play significant role in stress adaptation through scavenging of free- 
radical, storage of energy and osmoregulation. It is bio-synthesized from fructose in plants through the action of mannitol-1-phosphate dehydrogenase (mt1D). Its involvement in abiotic stress response was demonstrated through overexpressing a bacterial mannitol-1-phosphate dehydrogenase (mtlD) gene under control of the CaMV $35 \mathrm{~S}$ promoter conferred tolerance to various abiotic stresses including low temperature, drought, and salt stress to the transgenic tomato plants (Khare et al., 2010). The mt1D transgenic tomato plants showed significant reduction in electrolyte leakage, increased lipid peroxidation with enhancing antioxidant enzymes (SOD and catalase) and relative water content (RWC) compared to WT.

\subsection{Transcription Factors (TF) and Signaling Proteins}

Regulatory proteins such as transcription factors and signaling proteins are expected to modulate the expression of wide range of downstream genes involved in salt stress responses. Transcription factors such as ABRE, $\mathrm{MYC} / \mathrm{MYB}, \mathrm{CBF} / \mathrm{DREBs}, \mathrm{NAC}$ and WRKY have been genetically engineered to improve tolerance to abiotic stress in various crops (Wani et al., 2016). Members of different plant hormone response transcriptional factors also play important roles in regulating gene expression in response to biotic and abiotic stresses. For instance, constitutive expression of abscisic acid responsive element binding factor (AREB) conferred salt and drought tolerance in transgenic tomato through activating the expression of several stress associated genes (Orellana et al., 2010). Studies also demonstrated that the Ethylene Response Factor (EFR) TFs are involved in tomato salt stress tolerance. Klay et al. (2014) demonstrated that tomato Ethylene Response Factor (Sl-ERF.B.3) antisense transgenic plants exhibited a salt stress dependent growth inhibition or higher salt stress sensitivity compared to the WT. This inhibition was significantly enhanced in shoots but reduced in roots, leading to an increased root to shoot ratio. The dehydration responsive element binding proteins (DREBs) are other TFs, which are found only in plants and involved in the induction of abiotic stress-associated genes. A tomato dehydration-responsive element-binding 2 (SIDREB2) is identified as a salt stress regulated transcription factor (Hichri et al. 2016). Its overexpression in Arabidopsis and tomato improve plant tolerance to salinity. SIDREB2 enhanced plant tolerance to salinity by improving $\mathrm{K}^{+} / \mathrm{Na}^{+}$ratio, efficient regulation of protecting molecules such as proline, polyamines synthesis and hormonal contents, especially ABA. Moreover, it regulates the down-stream genes involved in stress response, osmoregulation and hormones biosynthesis/signaling. The tomato DREB1A (Dehydration-responsive elementbinding protein 1A) gene and AVP1 (a vacuolar $\mathrm{H}(+)$ - pyrophosphatase) are also shown to be associated with salt tolerance in Solanum pimpinellifolium (Rao et al., 2015).

$S L-Z H 13$ is one of the transcription factors reported to be involved in regulating tomato salt stress tolerance (Zhao et al., 2018). Silencing of $S L-Z H 13$ resulted in immediate wilting of the transgenic pants compared to the WT treated with similar salt concentration. The activities of superoxide dismutase, peroxidase, and proline content were lower in $S L-Z H 13$-silenced plants as superoxide dismutase, peroxidase, and proline content were lower in $S L-Z H 13$-silenced plants as compared to the WT plants after different time interval of salt treatment. Moreover, the accumulation of $\mathrm{H}_{2} \mathrm{O}_{2}$ and $\mathrm{O}_{2}$ - in leaves was much higher in SL-ZH13-silenced plants than control plants.

Plant-specific NAC transcription factors are reported to have significant role in response to abiotic stress. Zhu et al. (2014) examine the response of SINAC4-RNAi and WT tomato to salt and drought stress. The result demonstrated that SINAC4-RNAi transgenic tomato plants showed reduction in shoot and root growth as compared to the WT at post-germination stage. The SINAC4-RNAi transgenic lines were less tolerant to salt and drought with lower levels of water and chlorophyll contents, and higher rate of water loss from the leaves. Moreover, down-regulation of stress related genes in the transgenic line suggests a positive regulation of SINAC4 stressresponsive transcription factor to abiotic stress.

Similarly, the tomato ARS1 (altered response to salt stress 1) gene encodes a MYB transcription factor that contributes to reduced transpiration water loss under salt stress mediated by ABA (Campos et al., 2016).

WRKY proteins are another transcription factors, which are known to be involved in regulating diverse functional processes such as growth, development, hormone-mediated pathways, biotic and abiotic stresses (Ramamoorthy et al., 2008). They are a superfamily of transcription factors containing one or two highly conserved WRKY domains, 60 amino acid region comprising conserved WRKYGQK sequences at N-terminal together with zinc-finger-like motif at its C-terminal (Eulgem et al., 2000). The WRKY domain binds to W-box binding motif (T/CTGACC/T) of the target gene promoter region and activates or represses the expression of downstream genes. Compared to biotic stresses, the function of WRKY transcription factors in abiotic stresses is not well explored and thus not well understood. However, several studies showed that the expression of WRKY genes is induced by various environmental factors such as wounding, drought, salt, heat and cold stresses, and phytohormone treatments (Hara et al., 2000; Jiang and Deyholos, 2009; Wei et al., 2008).

Based on the genome wide investigation of WRKY genes in tomato, a total of 81 WRKY members were identified (Huang et al., 2012). Microarray analysis of these members showed significant change in expression pattern in response to biotic and abiotic stresses. Further expression analysis of selected SIWRKY in real-time quantitative RT-qPCR showed that majority of the tested SIWRKY genes were up-regulated in response to drought, salt, and pathogen invasion, while down regulation has also observed for some of the SIWRKY genes. Transgenic 
tomato overexpression SIWRKY3 improved multiple aspects of salinity tolerance at $125 \mathrm{mM} \mathrm{NaCl}$ (Hichri et al., 2017). Plant growth attributes such as shoot and root biomass and photosynthesis including stomatal conductance and chlorophyll a content were maintained in transgenic plants, which have lower $\mathrm{Na}^{+}$contents in leaves with higher accumulation of $\mathrm{K}^{+}$and $\mathrm{Ca}^{2+}$.

Moreover, several stress-related genes including genes coding for antioxidant enzymes, ion and water transporters were up-regulated in transgenic tomatoes as confirmed from microarray analysis. Similarly, SlWRKY39 enhanced resistance to multiple stress factors in tomato. Transgenic plants over-expressing SlWRKY39 improved resistance to multiple stress factors including PstDC3000, salt and drought (Sun et al., 2015). The transgenic plants showed accumulation of higher level of proline and lower malonic dialdehyde compared to the wild type due to up-regulation of genes related to environmental stress (e.g. SlRD22, SIDREB2A) and pathogenesis-related genes (SIPR1 and SIPRla1).

\section{Concluding remarks}

Salinity is one of the most important abiotic factors reducing agricultural crop production globally and is also expected to be a major challenge for the coming years. Efforts have been made both in model and other cultivated crops including tomato to improve salt stress through conventional breeding. However, the complex and multigenic nature of the trait makes low achievement in developing salt tolerance cultivars through traditional breeding. Biotechnological technique (genetic engineering) is a powerful tool in this regard to understand the molecular response mechanisms of salt stress and several successful results have been reported with regard to identification, isolation and cloning of genes involved in salt tolerance in tomato. Furthermore, the biotechnological technique helps to understand the molecular mechanisms of tolerance of the cloned and/or overexpressed candidate genes. Plasma membrane $\mathrm{Na}^{+} / \mathrm{H}^{+}$antiporters (SOS) and vacuolar $\mathrm{Na}^{+} / \mathrm{H}^{+}$antiporters (NHXn), Dehydrin (DHN), Choline Oxidase (codA), and ROS scavengers have been the major genes targeted in many studies for developing salt stress tolerant transgenic tomato. Moreover, promising results have reported on the identification and use of various abiotic stress responsive elements and/or transcription factors such as SIDREB2, SL-ZH13, Sl-ERF.B.3, AREB, NAC-Type and WRKY TFs, which regulate the expression of several downstream genes in transgenic tomato with response to salt stress. These transcription factors trigger cascades of gene expression that respond to various stress stimuli. Thus, modification of transcription factors could be an alternative option to enhance tolerance towards different environmental stress factors simultaneously. This is indeed a faster way and could be an option to reduce the growing global problem of salinity in major tomato producing areas.

\section{References}

- Achard P, Cheng H, De Grauwe L, Decat J, Schoutteten H, Moritz T, Van Der Straeten D, Peng J, Harberd NP (2006). Integration of plant responses to environmentally activated phytohormonal signals. Science 311 , 91-94.

- Acquaah G (2009). Principles of plant genetics and breeding Wiley. com.

- Albaladejo I, Plasencia FA, Meco V, Egea MI, Bolarin MC, Flores FB (2015). Different strategies used by domesticated tomato and wild-related species to confront salt stress. Procedia Environmental Sciences. 29:912.

- $\quad$ Apse MP, Aharon GS, Snedden WA, Blumwald E (1999). Salt tolerance conferred by overexpression of a vacuolar $\mathrm{Na}+/ \mathrm{H}+$ antiport in Arabidopsis. Science 285, 1256-1258.

- Ashraf M, Wu L (1994). Breeding for salinity tolerance in plants. Critical Reviews in Plant Sciences 13, $17-$ 42 .

- $\quad$ Borghesi E, González-Miret ML, Escudero-Gilete ML, Malorgio F, Heredia FJ, Meléndez-Martínez AJ (2011). Effects of salinity stress on carotenoids, anthocyanins, and color of diverse tomato genotypes. Journal of agricultural and food chemistry. 59(21):11676-82.

- Cuartero J, Fernández-Muñoz R (1998). Tomato and salinity. Scientia Horticulturae 78, 83-125.

- Cuartero J, Bolarin M, Asins M, Moreno V (2006). Increasing salt tolerance in the tomato. Journal of experimental botany 57, 1045-1058.

- $\quad$ Eulgem T, Rushton PJ, Robatzek S, Somssich IE (2000). The WRKY superfamily of plant transcription factors. Trends in plant science 5, 199-206.

- Foolad M (2004). Recent advances in genetics of salt tolerance in tomato. Plant Cell, Tissue and Organ Culture 76, 101-119.

- Gálvez FJ, Baghour M, Hao G, Cagnac O, Rodríguez-Rosales MP, Venema K (2012). Expression of LeNHX isoforms in response to salt stress in salt sensitive and salt tolerant tomato species. Plant Physiology and Biochemistry. 51:109-15.

- Gill SS, Tuteja N (2010). Reactive oxygen species and antioxidant machinery in abiotic stress tolerance in crop plants. Plant Physiology and Biochemistry 48, 909-930. 
- $\quad$ Gisbert C, Rus AM, Bolar MC, pe-Coronado n M L, Arrillaga I, Montesinos C, Caro M, Serrano R., Moreno V (2000). The yeast HAL1 gene improves salt tolerance of transgenic tomato. Plant physiology 123, 393-402.

- Hamayun M, Khan SA, Shinwari Z, Khan A, Ahmad N, Lee I (2010a). Effect of salt stress on growth attributes and endogenous growth hormones of soybean cultivar Hwangkeumkong. Pak. J. Bot 42, 3103-3112.

- Hamayun M, Khan SA, Khan AL, Shin J-H, Ahmad B, Shin D-H, Lee I-J (2010b). Exogenous gibberellic acid reprograms soybean to higher growth and salt stress tolerance. Journal of agricultural and food chemistry 58, 7226-7232.

- Hara K, Yagi M, Kusano T, Sano H (2000). Rapid systemic accumulation of transcripts encoding a tobacco WRKY transcription factor upon wounding. Molecular and General Genetics MGG 263, 30-37.

- Hasanuzzaman M, Nahar K, Fujita M, Ahmad P, Chandna R, Prasad M, Ozturk M (2013). Enhancing Plant Productivity Under Salt Stress: Relevance of Poly-omics Salt Stress in Plants, 113-156. Springer.

- Hichri I, Muhovski Y, Clippe A, Žižková E, Dobrev PI, Motyka V, Lutts S (2016). SIDREB2, a tomato dehydration-responsive element-binding 2 transcription factor, mediates salt stress tolerance in tomato and Arabidopsis. Plant, cell \& environment. 39(1):62-79.

- Hichri I, Muhovski Y, Zizkova E, Dobre P, Gharbi E, Lutts S (2017). The Solanum lycopersicum WRKY transcription factor SIWRKY3 is involved in salt stress tolerance in tomato. Frontiers in Plant Science. 8:18

- Huertas R, Olias R, Eljakaoui Z, GÁLVEZ FJ, Li J, DE MORALES PA, Belver A, RODRÍGUEZ-ROSALES MP (2012). Overexpression of SISOS2 (SICIPK24) confers salt tolerance to transgenic tomato. Plant, cell \& environment 35, 1467-1482.

- Iuchi S, Kobayashi M, Taji T, Naramoto M, Seki M, Kato T, Tabata S, Kakubari Y, Jaarsma K, de Vries R, RS, de Boer AH (2013). Effect of Salt Stress on Growth, Na+ Accumulation and Proline Metabolism in Potato (Solanum tuberosum) Cultivars. PloS one 8, e60183.

- Jiang Y, Deyholos MK (2009). Functional characterization of Arabidopsis NaCl-inducible WRKY25 and WRKY33 transcription factors in abiotic stresses. Plant molecular biology 69, 91-105.

- $\quad$ Khare N, Goyary D, Singh NK, Shah P, Rathore M, Anandhan S, Sharma D, Arif M, Ahmed Z (2010). Transgenic tomato cv. Pusa Uphar expressing a bacterial mannitol-1-phosphate dehydrogenase gene confers abiotic stress tolerance. Plant Cell, Tissue and Organ Culture (PCTOC). 103(2):267-77.

- $\quad$ Klay I, Pirrello J, Riahi L, Bernadac A, Cherif A, Bouzayen M, Bouzid S (2014). Ethylene response factor S1ERF. B. 3 is responsive to abiotic stresses and mediates salt and cold stress response regulation in tomato. The Scientific World Journal.

- Leem SC Luan S (2012). ABA signal transduction at the crossroad of biotic and abiotic stress responses. Plant, Cell \& Environment 35, 53-60.

- Leidi EO, Barragán V, Rubio L, El-Hamdaoui A, Ruiz MT, Cubero B, Fernández JA, Bressan RA, Hasegawa PM, Quintero FJ, Pardo JM (2010). The AtNHX1 exchanger mediates potassium compartmentation in vacuoles of transgenic tomato. Plant Journal 61, 495-506.

- $\quad$ Liu DD, Sun XS, Liu L, Shi HD, Chen SY, Zhao DK (2019). Overexpression of the Melatonin SynthesisRelated Gene SICOMT1 Improves the Resistance of Tomato to Salt Stress. Molecules 24(8):1514.

- $\quad$ Liu H, Yu C, Li H, Ouyang B, Wang T, Zhang J, Wang X, Ye Z (2015). Overexpression of ShDHN, a dehydrin gene from Solanum habrochaites enhances tolerance to multiple abiotic stresses in tomato. Plant Science. 231:198-211.

- Mandhania S, Madan S, Sawhney V (2006). Antioxidant defense mechanism under salt stress in wheat seedlings. Biologia Plantarum 50, 227-231.

- Munns R (2002). Comparative physiology of salt and water stress. Plant, Cell \& Environment 25, $239-250$.

- Munns R, Tester M (2008). Mechanisms of salinity tolerance. Annu. Rev. Plant Biol. 59, 651-681.

- Muñoz-Mayor A, Pineda B, Garcia-Abellán JO, Antón T, Garcia-Sogo B, Sanchez-Bel P, Flores FB, Atarés A, Angosto T, Pintor-Toro JA, Moreno V (2012). Overexpression of dehydrin tas 14 gene improves the osmotic stress imposed by drought and salinity in tomato. Journal of plant physiology. 169(5):459-68.

- Orellana S, Yanez M, Espinoza A, Verdugo I, Gonzalez E, RUIZ-LARA S, Casaretto JA (2010). The transcription factor SIAREB1 confers drought, salt stress tolerance and regulates biotic and abiotic stressrelated genes in tomato. Plant, cell \& environment 33, 2191-2208.

- Orsini F, Alnayef M, Bona S, Maggio A, Gianquinto G (2012). Low stomatal density and reduced transpiration facilitate strawberry adaptation to salinity. Environmental and Experimental Botany 81, 1-10.

- Ramamoorthy R, Jiang S-Y, Kumar N, Venkatesh PN, Ramachandran S (2008). A comprehensive transcriptional profiling of the WRKY gene family in rice under various abiotic and phytohormone treatments. Plant and cell physiology 49, 865-879.

- $\quad$ Rao ES, Kadirvel P, Symonds RC, Geethanjali S, Thontadarya RN, Ebert AW (2015). Variations in DREB1A and VP1. 1 genes show association with salt tolerance traits in wild tomato (Solanum pimpinellifolium). PloS 
one. 10(7):e0132535.

- $\quad$ Rebah F, Ouhibi C, Alamer KH, Msilini N, Nasri MB, Stevens R, Attia H (2018). Comparison of the responses to $\mathrm{NaCl}$ stress of three tomato introgression lines. Acta Biologica Hungarica 69(4):464-80.

- Rengasamy P (2010). Soil processes affecting crop production in salt-affected soils. Functional Plant Biology 37, 613-620.

- Robertson LD, Labate JA (2007). Genetic resources of tomato (Lycopersicon esculentum Mill.) and wild relatives. Genetic improvement of Solanaceous crops, 25-75.

- Rodríguez-Rosales MP, Jiang X, Gálvez FJ, Aranda MN, Cubero B, Venema K (2008). Overexpression of the tomato $\mathrm{K}+/ \mathrm{H}+$ antiporter LeNHX2 confers salt tolerance by improving potassium compartmentalization. New Phytologist. 179(2):366-77.

- $\quad$ Shi H, Lee B-h, Wu S-J, Zhu J-K (2002a). Overexpression of a plasma membrane $\mathrm{Na}+\mathrm{H}+$ antiporter gene improves salt tolerance in Arabidopsis thaliana. Nature biotechnology 21, 81-85.

- Shi H, Quintero FJ, Pardo JM, Zhu J-K (2002b). The putative plasma membrane Na+/H+ antiporter SOS1 controls long-distance $\mathrm{Na}+$ transport in plants. The Plant Cell Online 14, 465-477.

- Sottosanto JB, Saranga Y, Blumwald E (2007): Impact of AtNHX1, a vacuolar Na+/H+ antiporter, upon gene expression during short-and long-term salt stress in Arabidopsis thaliana. BMC plant biology 7, 18.

- Sripinyowanich S, Klomsakul P, Boonburapong B, Bangyeekhun T, Asami T, Gu H, Buaboocha T, Chadchawan S (2010). Exogenous ABA induces salt tolerance in indica rice $(<\mathrm{i}>$ Oryza sativa $</ \mathrm{i}>\mathrm{L}$. $)$ : The role of $<\mathrm{i}>\operatorname{OsP} 5 \mathrm{CS} 1</ \mathrm{i}>$ and $<\mathrm{i}>\mathrm{OsP} 5 \mathrm{CR}</ \mathrm{i}>$ gene expression during salt stress. Environmental and Experimental Botany.

- Szabolcs I (1989). Salt-affected soils CRC Press, Inc.

- Uozumi N, Kim EJ, Rubio F, Yamaguchi T, Muto S, Tsuboi A, Bakker EP, Nakamura T, Schroeder JI (2000). The Arabidopsis HKT1 gene homolog mediates inward Na+ currents in Xenopus laevis oocytes and $\mathrm{Na}+$ uptake in Saccharomyces cerevisiae. Plant physiology 122, 1249-1260.

- Vinocur B, Altman A (2005). Recent advances in engineering plant tolerance to abiotic stress: achievements and limitations. Current opinion in biotechnology. 16(2):123-32.

- Viveros MF, Inostroza-Blancheteau C, Timmermann T, González M, Arce-Johnson P (2013). Overexpression of GlyI and GlyII genes in transgenic tomato (Solanum lycopersicum Mill.) plants confers salt tolerance by decreasing oxidative stress. Molecular biology reports. 40(4):3281-90.

- Vranova E, Inzé D, Van Breusegem F (2002). Signal transduction during oxidative stress. Journal of experimental botany 53, 1227-1236.

- Wani SH, Sah SK, Hossain MA, Kumar V, Balachandran SM (2016). Transgenic approaches for abiotic stress tolerance in crop plants. In Advances in Plant Breeding Strategies: Agronomic, Abiotic and Biotic Stress Traits (pp. 345-396). Springer, Cham.

- Wei D, Zhang W, Wang C, Meng Q, Li G, Chen TH, Yang X (2017). Genetic engineering of the biosynthesis of glycinebetaine leads to alleviate salt-induced potassium efflux and enhances salt tolerance in tomato plants. Plant science. 257:74-83.

- Wei W, Zhang Y, Han L, Guan Z, Chai T (2008). A novel WRKY transcriptional factor from Thlaspi caerulescens negatively regulates the osmotic stress tolerance of transgenic tobacco. Plant cell reports 27, 795803.

- Xia T, Apse MP, Aharon GS, Blumwald E (2002). Identification and characterization of a NaCl-inducible vacuolar $\mathrm{Na}+/ \mathrm{H}+$ antiporter in Beta vulgaris. Physiologia Plantarum 116, 206-212.

- Yada RC, Solanke AU, Kumar P, Pattanayak D, Yadav NR, Kumar PA (2013). Genetic Engineering for Tolerance to Climate Change-Related Traits Genomics and Breeding for Climate-Resilient Crops, Springer 285-330.

- Yarra R, He SJ, Abbagani S, Ma B, Bulle M, Zhang WK (2012a). Overexpression of a wheat Na +/H + antiporter gene (TaNHX2) enhances tolerance to salt stress in transgenic tomato plants (Solanum lycopersicum L.). Plant Cell, Tissue and Organ Culture 111, 49-57.

- Yang J, Cao Y, Yang Z, Zhang W, Sun L, Lu C (2013). Morphological, physiological and biochemical responses of biofuel plant Euphorbia lathyris to salt stress. Acta Agriculturae Scandinavica, Section B-Soil \& Plant Science 63, 330-340.

- Zhang, H-X, and Blumwald E (2001). Transgenic salt-tolerant tomato plants accumulate salt in foliage but not in fruit. Nature biotechnology 19, 765-768.

- Zhao T, Hu J, Gao Y, Wang Z, Bao Y, Zhang X, Yang H, Zhang D, Jiang J, Zhang H, Li J (2018). Silencing of the SL-ZH13 Transcription Factor Gene Decreases the Salt Stress Tolerance of Tomato. Journal of the American Society for Horticultural Science. 143(5):391-6.

- Zhu J-K (2001). Plant salt tolerance. Trends in plant science 6, 66-71. 
- Zhu M, Chen G, Zhang J, Zhang Y, Xie Q, Zhao Z, Pan Y, Hu Z (2014). The abiotic stress-responsive NACtype transcription factor SINAC4 regulates salt and drought tolerance and stress-related genes in tomato (Solanum lycopersicum). Plant cell reports. 33(11):1851-63. 